\title{
Timer-based Demodulator for AM Sensor Signals applied to an Inductive Displacement Sensor
}

\author{
Ferran Reverter and Manel Gasulla
}

\begin{abstract}
This paper proposes a novel method for demodulating low-frequency amplitude-modulated (AM) signals provided by sensors. The method relies on a digital timer that carries out the demodulation and digitization simultaneously, without requiring a rectifier, a mixer, a low-pass filter, or an analog-to-digital converter. This timer-based demodulator extracts the amplitude of the AM signal by measuring the period of a reference signal that is altered by the AM signal itself. The period measurement undergoes a deviation that carries information about the amplitude of the AM signal. The feasibility and also the limitations, such as the nonlinearity and aliasing effects, of this novel demodulator are proved theoretically and experimentally. The concept is also applied to measure an inductive displacement sensor in a range of $\pm 30 \mathrm{~mm}$. A non-linearity error of $0.5 \%$ fullscale span and a resolution of 9 bits are achieved for an overall measuring time of $100 \mathrm{~ms}$.
\end{abstract}

Index Terms- Demodulator, digital timer, displacement sensor, inductive sensor, sensor interface electronics.

\section{INTRODUCTION}

$\mathrm{I}$ $\mathrm{N}$ the field of electronic instrumentation, many sensors are read by a signal conditioning circuit that excites the sensor through an alternating (AC) signal. This is the case for capacitive and inductive sensors, which are widely employed to measure displacement [1], flow rate [2], human posture [3], position, level, among others. Resistive sensors based on electrolytes also require an $\mathrm{AC}$ excitation so as to avoid electrolysis. As a result of this AC excitation, the variation of resistance, capacitance, or inductance modulates the amplitude (AM) or the frequency (FM) of an alternating output signal. This output signal is generally sinusoidal for the AM case, as happens in AC bridges [4-6], but it can be sinusoidal or square for the FM case, as occurs in harmonic and relaxation oscillators [7], respectively.

In AM sinusoidal signals, the amplitude is modulated by the

Manuscript received Month $x x, 2 x x x$; revised Month $x x, x x x x$ accepted Month $\mathbf{x}, \mathbf{x x x x}$. This work was supported by the Spanish Ministry of Economy and Competitiveness and the European Regional Development Fund under project TEC2016-76991-P.

Ferran Reverter and Manel Gasulla are with the Department of Electronic Engineering, Universitat Politècnica de Catalunya BarcelonaTech, Castelldefels (Barcelona), 08860, Spain (e-mail: ferran.reverter@upc.edu; manel.gasulla@upc.edu). measurand, whereas the carrier frequency is constant. This frequency is usually equal to units of kilohertz for inductive sensors [2,4-6], and tens [8,9] or hundreds [10,11] of kilohertz for capacitive sensors. The demodulation of these signals can be either asynchronous or synchronous. An envelope detector, which is the most employed asynchronous demodulator, has a rectifier and a peak detector that can be a simple RC-circuit $[2,5,11]$ or a more advanced design providing shorter time constants [12,13]. On the other hand, a synchronous demodulator relies on a mixer and a low-pass filter (LPF) $[3,8,10]$. For double-sideband transmitted carrier AM signals, as happens in $\mathrm{AC}$ voltage dividers, the demodulation can be asynchronous or synchronous, but taking into account that the latter offers a higher capacity to reject noise/interference [14] at the expense of a more complex circuit. However, for double-sideband suppressed carrier AM signals, as occurs in AC bridges, the demodulation must be synchronous so as to detect phase changes. Whatever the case, after demodulating the signal in the analog domain, the resulting low-frequency signal is digitized by an analog-to-digital converter (ADC). The demodulation can also be carried out in the digital domain but using first an ADC operating at a higher sampling frequency $[6,15]$.

In FM sensor signals, the frequency or the period is modulated by the measurand, whereas the amplitude is constant; for instance, it is equal to the supply voltage of the oscillator in FM square signals. This type of modulation has the advantage that the dynamic range is not limited by the supply voltage of the circuit. The main drawback is that the sensor is not excited at a constant frequency and, hence, the sensor specifications given at a nominal frequency can be inapplicable along all the frequency range. The demodulation of these signals can be carried out by a time-to-digital converter (TDC), which can be implemented by a digital timer/counter $[16,17]$. Sensor circuits based on TDCs, instead of ADCs, have advantages in terms of current consumption and layout area. Note, for instance, that in recent low-cost mixed-signal microcontrollers, the more power-demanding peripheral is the ADC, with a current consumption of hundreds of microampere. On the other hand, in integrated designs, a digital timer/counter can occupy a layout area smaller than $0.01 \mathrm{~mm}^{2}[18,19]$, which is one or two orders of magnitude smaller than that required by a successiveapproximation [20] or a delta-sigma ADC [21], respectively. 
Taking into account the advantages of using TDCs instead of ADCs, extensive research has been carried out in the development of timer-based conditioning circuits for resistive [22-24], capacitive [25-27], and inductive sensors [28,29]. Most of these circuits rely on measuring the charging or discharging time of a first-order circuit that is excited by a step of voltage. Sensors with a quasi-static analog output voltage have also been measured using a timer-based conditioning circuit [30]. However, the measurement or the demodulation of AM sinusoidal signals by means of a digital timer has never been suggested before in the literature.

This paper proposes a novel method for the demodulation of AM sinusoidal signals using a digital timer, which can be embedded into a low-cost microcontroller, as a core and without requiring the typical blocks of an AM demodulator. This is intended for AM signals with a carrier frequency in the range of kilohertz, as happens in circuits for inductive and capacitive sensors, and without carrier suppression. The demodulation of signals with a higher carrier frequency (say, higher than $1 \mathrm{MHz}$ [31]) is out of the scope of the proposed demodulator since it would require a timer with a crystal oscillator of very high frequency.

The paper is organized as follows. Section II qualitatively describes the operating principle of the proposed demodulator. Section III theoretically analyzes its performance and limitations. Section IV shows experimental results and Section $\mathrm{V}$ applies the concept to demodulate the signal coming from an inductive displacement sensor. Section VI draws the main conclusions. An Appendix analyses the aliasing phenomenon involved in the proposed measurement method.

\section{OPERATING PRINCIPLE}

The proposed timer-based demodulator extracts the amplitude of the AM sinusoidal signal by measuring the period of a reference triangular signal, which suffers from interference effects caused by the AM signal itself. These concepts are explained in more detail next.

\section{A. Period measurement}

The period measurement of a signal through a digital timer relies on the following principle. When the input signal crosses a given threshold voltage, the digital timer starts counting high-frequency pulses coming from a crystal oscillator. After one period of the input signal, this crosses again the same threshold voltage with the same edge and the digital timer stops. The instants at which the timer starts and stops are identified next as starting and stopping trigger points, respectively. The period of the signal can be then calculated as the (digital) number of counts registered in the timer multiplied by the period of the oscillator signal. This measurement suffers from three main uncertainty sources [32]: instability of the crystal oscillator, quantization, and trigger noise/interference. The effects of the latter are here exploited to demodulate the AM sinusoidal signal, which will be superimposed on the threshold voltage to intentionally alter the trigger points during the period measurement of a reference signal.

\section{B. Interference effects on period measurements}

If the method explained before is applied to measure the period of a signal with a slow slew rate, the measurement becomes susceptible to interference superimposed on both the threshold voltage and the input signal [33]. Both the starting and stopping trigger points are erroneous due to the interference and, therefore, the period measurement suffers from a non-constant deviation. This variability, which can be quantified by the standard deviation (STD) of the measurements, increases with the amplitude of the interference and depends on the ratio between the interference frequency $\left(f_{\mathrm{i}}\right)$ and the input-signal frequency $\left(f_{0}\right)$. Interference effects are null when $f_{\mathrm{i}} / f_{0}=m$, whereas they are maximum when $f_{\mathrm{i}} / f_{0}=m+0.5, m$ being any positive integer including the zero [33]. Such a performance is similar to what happens

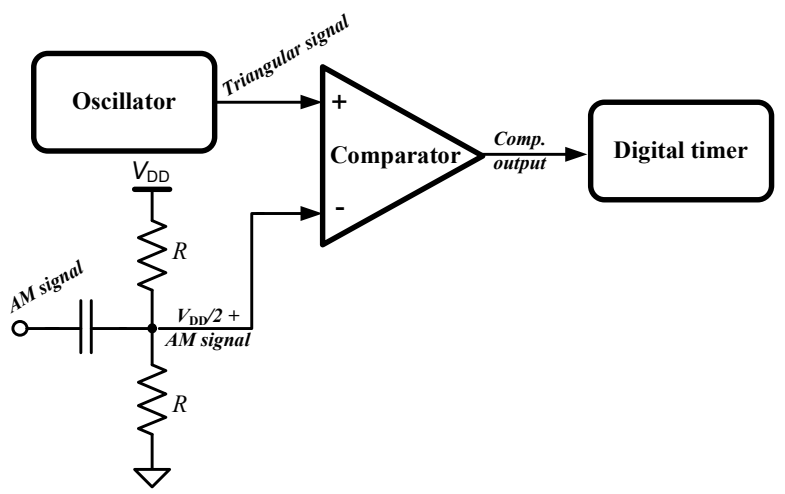

Fig. 1. Proposed demodulator for AM signals based on a digital timer.

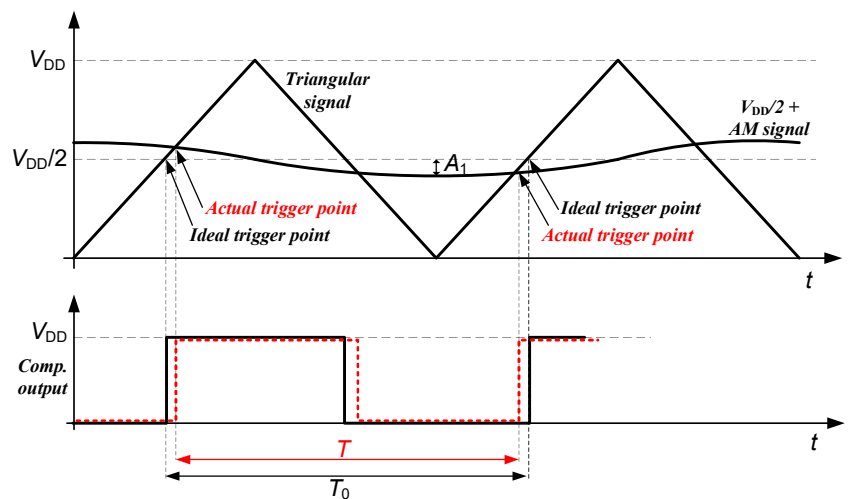

Fig. 2. Waveform of the main signals in Fig. 1 when the AM signal has an amplitude $A_{1}$

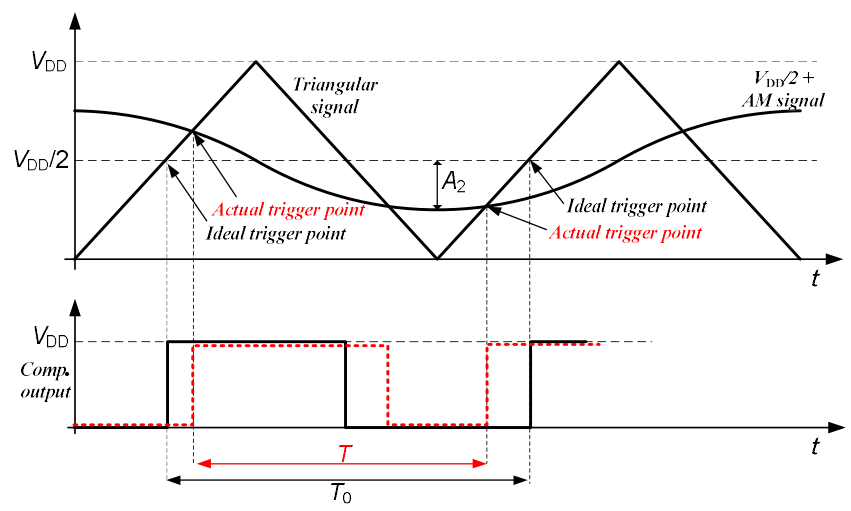

Fig. 3. Waveform of the main signals in Fig. 1 when the AM signal has an amplitude $A_{2}$ 
in integrating ADCs that completely reject interference superimposed on the input signal provided that the period of the interference is a submultiple of the integrating time of the ADC. In the proposed demodulator, the AM signal will behave as interference during the period measurement.

\section{C.AM demodulation based on period measurements}

The AM signal is proposed to be demodulated by superimposing it on the threshold voltage during the period measurement of a reference triangular signal. Fig. 1 shows the basic circuit to carry out such an operating principle. The AM signal is AC-coupled through a capacitor to a resistive voltage divider. Assuming two equal resistors, the resulting voltage is $V_{\mathrm{DD}} / 2$ plus the AM signal, which will act as a threshold voltage. On the other hand, an oscillator provides a triangular signal with an amplitude of $V_{\mathrm{DD}}$, an offset of $V_{\mathrm{DD}} / 2$, and a period of $T_{0}$. Both signals are then compared via a comparator that provides at the output a square signal whose period is measured by a digital timer.

If the amplitude of the AM signal is zero, the threshold voltage is free of interference and, therefore, the comparison between the two signals is triggered at the ideal trigger points. In these conditions, the comparator output is a square signal with a period equal to $T_{0}$. However, if the amplitude of the AM signal is $A_{1}$, the comparison is triggered at erroneous trigger points, as shown in Fig. 2, thus resulting in a square signal with a period equal to $T$ instead of $T_{0}$. A higher amplitude of the AM signal (i.e. $A_{2}>A_{1}$ ) generates a higher deviation in the period measurement, as shown in Fig. 3 assuming that the phase shift between the triangular and sinusoidal signals is the same considered before in Fig. 2. The measurement of $N$ periods in such conditions generates $N$ different values of $T$, each with a particular deviation with respect to the ideal value. The root mean square (RMS) value of the set of deviations is expected to increase with increasing the amplitude of the AM signal.

\section{THEORETICAL ANALYSIS}

The proposed demodulator aims to extract the amplitude of the following sinusoidal signal:

$$
v(t)=\frac{A_{\mathrm{s}}}{2} \sin \left(2 \pi f_{\mathrm{c}} t+\varphi\right)
$$

where $A_{\mathrm{s}}$ is the peak-to-peak amplitude modulated by the measurand, $f_{\mathrm{c}}$ is the carrier frequency, and $\varphi$ is the phase shift with respect to the triangular signal. If the signal described by (1) is superimposed on the threshold voltage when the period of a triangular signal is measured, the starting trigger point is moved from $t_{1}{ }^{*}$ to $t_{1}$, whereas the stopping trigger point from $t_{2}{ }^{*}$ to $t_{2}$, as shown in Fig. 4. Consequently, at both trigger points, there is a time error or deviation that can expressed as

$$
\begin{gathered}
e_{1}=-v\left(t_{1}\right) / \mathrm{SR} \\
e_{2}=v\left(t_{2}\right) / \mathrm{SR}
\end{gathered}
$$

where $v\left(t_{1}\right)$ and $v\left(t_{2}\right)$ are the voltage values of (1) at the actual trigger points $t_{1}$ and $t_{2}$, respectively, and $\mathrm{SR}\left(=2 V_{\mathrm{DD}} / T_{0}\right)$ is the

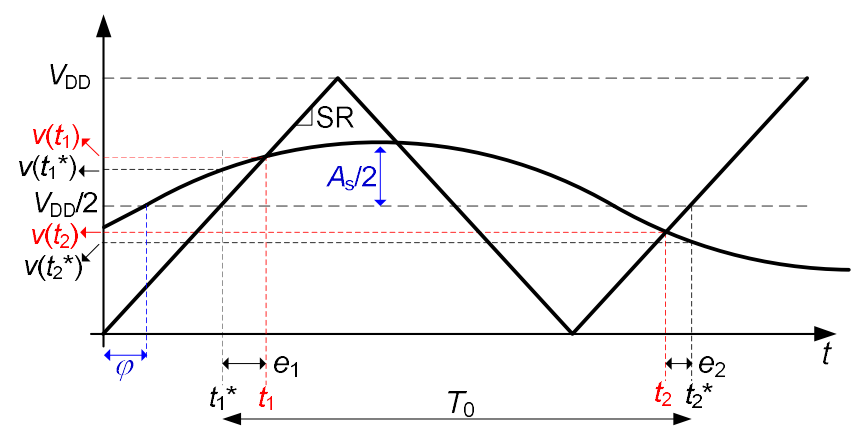

Fig. 4. Deviation $\left(e_{1}\right.$ and $\left.e_{2}\right)$ generated by the AM signal during the period measurement of a triangular signal.

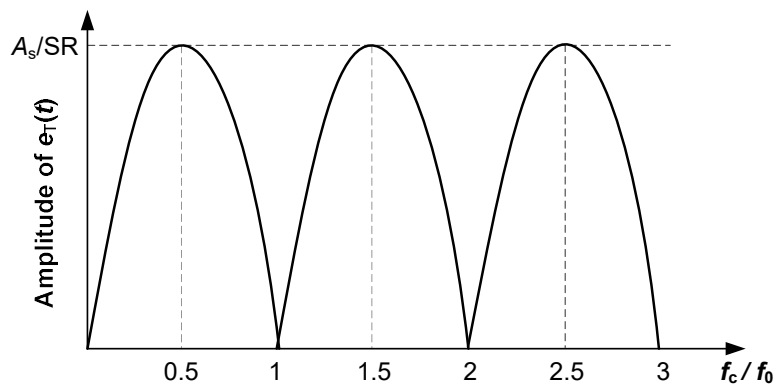

Fig. 5. Amplitude of the overall deviation $\left(e_{T}\right)$ in the period measurement versus the ratio $f_{c} / f_{0}$

slew rate of the triangular signal. The deviation is assumed positive if it increases the value of the measured period; accordingly, both deviations represented in Fig. 4 are negative. This sign convention involves the minus sign included in (2a). The overall deviation in the period measurement can be then calculated, from (2), as

$$
e_{\mathrm{T}}=e_{1}+e_{2}=\frac{v\left(t_{2}\right)-v\left(t_{1}\right)}{\mathrm{SR}}
$$

\section{A. Small-signal model}

For a low-amplitude low-frequency AM signal, the following approximation can be assumed [34]

$$
\begin{aligned}
& v\left(t_{1}\right) \approx v\left(t_{1}^{*}\right) \\
& v\left(t_{2}\right) \approx v\left(t_{2}^{*}\right)
\end{aligned}
$$

where $v\left(t_{1}{ }^{*}\right)$ and $v\left(t_{2}^{*}\right)$ are the voltage values of (1) at the ideal trigger points $t_{1}{ }^{*}$ and $t_{2}{ }^{*}$, respectively. Replacing (4) in (3) and considering $t_{2}{ }^{*}$ to be the time 'now' and $t_{1}{ }^{*}$ the time $T_{0}$ 'ago' [34] yields

$$
e_{\mathrm{T}}(t) \approx \frac{v(t)-v\left(t-T_{0}\right)}{\mathrm{SR}}
$$

Substituting now (1) in (5) gives

$$
e_{\mathrm{T}}(t)=\frac{A_{\mathrm{s}}}{\mathrm{SR}} \sin \left(\pi \frac{f_{\mathrm{c}}}{f_{0}}\right) \cos \left(2 \pi f_{\mathrm{c}} t+\varphi-\pi \frac{f_{\mathrm{c}}}{f_{0}}\right)
$$

where $f_{0}=1 / T_{0}$. According to (6), the deviation in the period measurement evolves as a sinusoidal signal with an amplitude 
and phase that depend on the ratio $f_{\mathrm{c}} / f_{0}$. The amplitude is zero when $f_{\mathrm{c}} / f_{0}=m$, whereas it is maximum when $f_{\mathrm{c}} / f_{0}=m+0.5$, $m$ being any positive integer including the zero, as shown in Fig. 5. For $f_{\mathrm{c}} / f_{0}=m+0.5$, which is here of interest so as to have the highest effects of the AM signal on the period measurement, (6) can be simplified to

$$
e_{\mathrm{T}}(t)=\frac{2}{\mathrm{SR}} v(t)
$$

which has an amplitude that is proportional to the amplitude of the AM signal.

The use of an exponential signal resulting from the chargedischarge process of an $\mathrm{RC}$ circuit instead of a triangular signal is in principle not recommended. This is because then the SR is not constant, thus generating a non-linear relation between the amplitude of $e_{\mathrm{T}}$ and the amplitude of the AM signal, even in a small-signal model.

\section{B. Large-signal model}

The approximation considered in (4) has several limitations if the amplitude of the AM signal is high (say, in the range of units of volt). To analyze the response of the timer-based demodulator for such values of amplitude, we propose to express $v\left(t_{1}\right)$ and $v\left(t_{2}\right)$ with a second-order Taylor-series approximation:

$$
\begin{aligned}
& v\left(t_{1}\right) \approx v\left(t_{1}^{*}\right)-\frac{d v\left(t_{1}^{*}\right)}{d t} e_{1}+\frac{1}{2} \frac{d^{2} v\left(t_{1}^{*}\right)}{d t^{2}} e_{1}^{2} \\
& v\left(t_{2}\right) \approx v\left(t_{2}^{*}\right)+\frac{d v\left(t_{2}^{*}\right)}{d t} e_{2}+\frac{1}{2} \frac{d^{2} v\left(t_{2}^{*}\right)}{d t^{2}} e_{2}^{2}
\end{aligned}
$$

The minus sign in the second term in $(8 \mathrm{a})$ is necessary to compensate for the minus sign of $e_{1}$ when $t_{1}>t_{1}{ }^{*}$, following the sign convention indicated before. In addition, for the case of interest in which $f_{\mathrm{c}} / f_{0}=m+0.5$, we have the following relations:

$$
\begin{gathered}
v\left(t_{2}^{*}\right)=-v\left(t_{1}^{*}\right) \\
\frac{d v\left(t_{2}^{*}\right)}{d t}=-\frac{d v\left(t_{1}^{*}\right)}{d t} \\
\frac{d^{2} v\left(t_{2}^{*}\right)}{d t^{2}}=-\frac{d^{2} v\left(t_{1}^{*}\right)}{d t^{2}}
\end{gathered}
$$

Therefore, substituting (9) in (8a), (8) can be rewritten in function of $t_{2}{ }^{*}$ as

$$
\begin{aligned}
& v\left(t_{1}\right) \approx-v\left(t_{2}^{*}\right)+\frac{d v\left(t_{2}^{*}\right)}{d t} e_{1}-\frac{1}{2} \frac{d^{2} v\left(t_{2}^{*}\right)}{d t^{2}} e_{1}^{2} \\
& v\left(t_{2}\right) \approx v\left(t_{2}^{*}\right)+\frac{d v\left(t_{2}^{*}\right)}{d t} e_{2}+\frac{1}{2} \frac{d^{2} v\left(t_{2}^{*}\right)}{d t^{2}} e_{2}^{2}
\end{aligned}
$$

Replacing now (10a) in (2a) and (10b) in (2b) yields two quadratic equations in the unknowns $e_{1}$ and $e_{2}$, respectively. Solving these and adding the results, we can find an

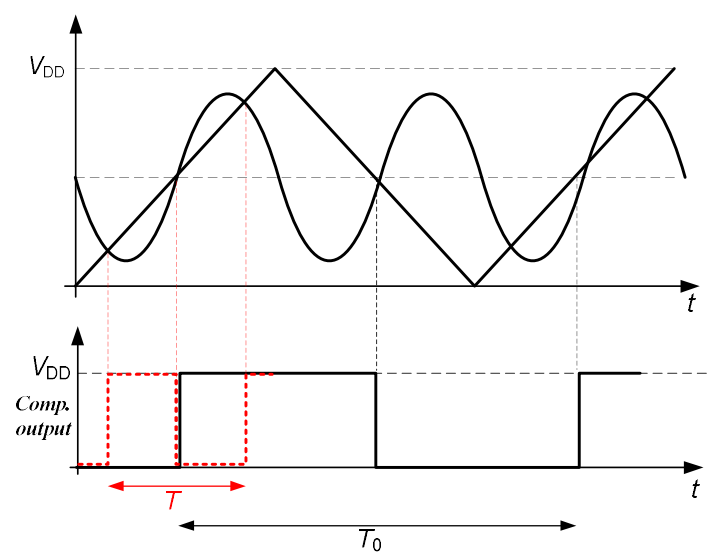

Fig. 6. Waveform of the main signals in Fig. 1 when the amplitude of the AM signal is too high, thus producing an aberrant period measurement of the triangular signal.

expression of $e_{\mathrm{T}}$ that can be written, assuming again $t_{2}{ }^{*}$ to be the time 'now', as

$$
e_{\mathrm{T}}(t)=\underbrace{\frac{2}{\mathrm{SR}} v(t)}_{\text {Term } 1} \underbrace{\left[\sqrt{y^{2}(t)+2 y(t)}-y(t)\right]}_{\text {Term } 2}
$$

where

$$
y(t)=\left(\frac{\mathrm{SR}}{2 \pi f_{\mathrm{c}} v(t)}\right)^{2}
$$

For low values of $A_{\mathrm{s}}, y(t)$ becomes a high $(>>1)$ timedependent value so that Term 2 in (11) can be simplified to 1 , except for the instants corresponding to the half and full period of $v(t)$ at which Term 2 is equal to 0 . Therefore, (11) can be simplified to Term 1, which is the same result as in the small-signal analysis [see (7)]. However, for high values of $A_{\mathrm{s}}$, $y(t)$ is a low time-dependent value and Term 2 in (11) becomes smaller than 1 and time-dependent, thus attenuating and distorting the sinusoidal signal described by Term 1 . Accordingly, the relation between the amplitude of $e_{\mathrm{T}}$ and the amplitude of the AM signal is expected to be non-linear, especially at high values of $A_{\mathrm{s}}$.

In addition to the non-linearity effects indicated before, the proposed method can suffer from aberrant period measurements if the amplitude of the AM signal is too high. This is qualitatively shown in Fig. 6, where a high-amplitude AM signal causes non-expected crossings between the two signals under comparison, thus resulting in an aberrant period measurement ( $T$ instead of $T_{0}$ ). In order to avoid this, the slope (or SR) of the triangular signal must be higher than the maximum slope of the AM signal, which involves

$$
A_{\mathrm{s}}<\frac{2 V_{\mathrm{DD}}}{\pi} \frac{f_{0}}{f_{\mathrm{c}}}
$$

Assuming $V_{\mathrm{DD}}=5 \mathrm{~V}$, the maximum value of $A_{\mathrm{s}}$ resulting from (13) is $6.37 \mathrm{~V}, 2.12 \mathrm{~V}$, and $1.27 \mathrm{~V}$ for $f_{\mathrm{c}} / f_{0}=0.5,1.5$, and 2.5 , respectively. 


\section{C.Sampling and aliasing}

According to (7) and (11), if the period measurement is subjected to the effects of the AM sinusoidal signal, then the resulting deviation is also sinusoidal (distorted if $A_{\mathrm{s}}$ is high) with the same frequency $f_{\mathrm{c}}$ and an amplitude that depends on $A_{\mathrm{s}}$ and SR. The analysis method developed in [34] states that a sample of this sinusoidal signal is taken every time the period of the triangular signal is measured. In a demodulator based on a single digital timer, the period can be measured at most every other cycle; note that the timer cannot end and start simultaneously on the same cycle edge. In such a case, which is then tested experimentally in Section IV, the sinusoidal signal represented by (7) or (11) is sampled at a frequency $\left(f_{\mathrm{s}}\right)$ that is half the frequency of the triangular signal, i.e. $f_{\mathrm{s}}=f_{0} / 2$. In a demodulator based on two digital timers operating in parallel, it would be possible to carry out back-to-back period measurements, thus resulting in $f_{\mathrm{s}}=f_{0}$.

If $f_{\mathrm{c}} / f_{0}=m+0.5$ and $f_{\mathrm{s}}=f_{0} / 2$, then $f_{\mathrm{s}}=f_{\mathrm{c}} /(1+2 m)$. For example, $f_{\mathrm{s}}=f_{\mathrm{c}}, f_{\mathrm{c}} / 3$, and $f_{\mathrm{c}} / 5$ for $m=0,1$, and 2 , respectively. Consequently, the Nyquist criterion, which states that the signal should be sampled at $f_{\mathrm{s}} \geq 2 f_{\mathrm{c}}$, is not satisfied. In these conditions, an alias component at $0 \mathrm{~Hz}$ is generated, as demonstrated in the Appendix. An alias component of $0 \mathrm{~Hz}$ means that the same value of $e_{\mathrm{T}}$ is repeatedly sampled and, therefore, a set of period measurements does not provide useful information to estimate the amplitude of the AM signal.

The previous limitation can be avoided by running the demodulator at $f_{\mathrm{c}} / f_{0}=(m+0.5)(1+\Delta)$, where $|\Delta|<<1$. Then, the sampling frequency is slightly shifted to

$$
f_{\mathrm{s}}=\frac{f_{\mathrm{c}}}{(1+2 m)(1+\Delta)}
$$

which generates a low-frequency alias component at $f_{\mathrm{c}} \cdot|\Delta|$, as demonstrated in the Appendix, that is independent of $m$. The amplitude of this alias can be considered equal to that of the original signal described by (7) or (11) provided that $|\Delta|<<1$, as shown in (A.4). Therefore, although the wave reconstructed from the period samples has a frequency different to $f_{\mathrm{c}}$, its amplitude has information about the amplitude of the AM signal of interest.

\section{D.Processing}

In order to correctly characterize the discrete-time signal resulting from the sampling process, it is necessary to take samples of the period of the triangular signal at least during one period of the alias component, i.e. $\left(f_{\mathrm{c}} \cdot|\Delta|\right)^{-1}$. This can be considered the overall measuring time since the processing can be quite fast in current digital systems. An alias component of a higher frequency (obtained with a higher value of $\Delta$ ) decreases the measuring time but with the following limitations: (i) the number of samples (per period of the alias component) becomes smaller and, hence, the characterization of the discrete-time signal could be more complicated; and (ii) the approximation $f_{\mathrm{c}} / f_{0} \approx m+0.5$ becomes less valid and, therefore, the amplitude of $e_{\mathrm{T}}$ would be lower, as can be observed in Fig. 5 and in (A.4).

Once the samples of the triangular-signal period have been taken during one period of the alias component, we propose to

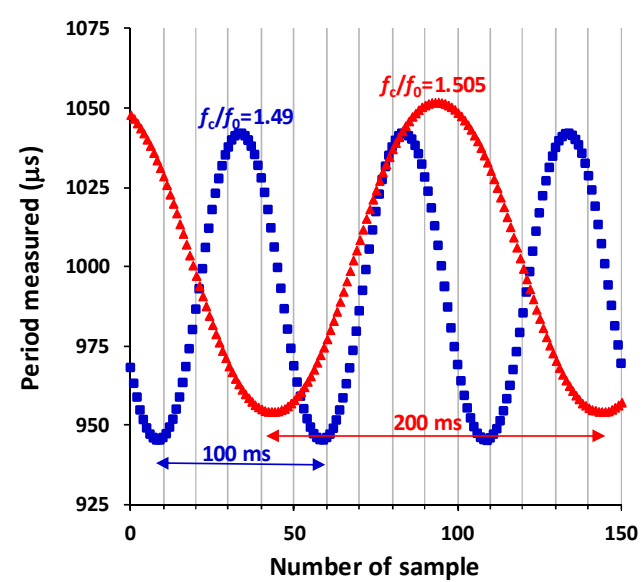

Fig. 7. Period samples obtained for $A_{s}=500 \mathrm{mV}$. Two cases with $f_{\mathrm{c}} / f_{0} \approx 1.5$ involving different alias components are represented. Assuming $f_{\mathrm{s}}=f_{0} / 2$, a sample of period was taken every $2 \mathrm{~ms}$.

process them through the STD. This provides the RMS level of the AC component of the period samples, or in other words: the RMS of $e_{\mathrm{T}}$. Another option would be to find the maximum value of the period samples so as to estimate the amplitude of $e_{\mathrm{T}}$. However, this method could be more sensitive to noise since it would rely on the value of a single sample (i.e. the maximum value).

\section{EXPERIMENTAL RESULTS AND DISCUSSION}

\section{A. Materials and method}

The concept of timer-based demodulator shown in Fig. 1 has been experimentally proved using the following setup. A waveform generator (Agilent 33120A) provided the triangular signal with an amplitude of $5 \mathrm{~V}$, an offset of $2.5 \mathrm{~V}$, and a frequency of $f_{0}$. Another waveform generator (Agilent $33210 \mathrm{~A}$ ) provided the AM sinusoidal signal at $f_{\mathrm{c}}=1500 \mathrm{~Hz}$, which is in the range of typical exciting frequencies for inductive sensors [4-6]. The different ratios of $f_{\mathrm{c}} / f_{0}$ under test were obtained by changing $f_{0}$ and keeping $f_{\mathrm{c}}$ constant. These two waveform generators were not synchronized so that the phase shift between the two generated signals was arbitrary for each set of period measurements. The AM signal also had an offset level of $2.5 \mathrm{~V}$ and, hence, the capacitor and the two resistors shown in Fig. 1 were not necessary. These two signals were compared using an ultrafast single-supply comparator (Analog Devices AD8561). The period of the comparator output was then measured using a bench-top universal counter (Agilent 53220A). This was configured to carry out a single-period measurement, which was realized every other cycle according to the internal firmware of the instrument. Consequently, as indicated before, $f_{\mathrm{s}}=f_{0} / 2$.

\section{B. Preliminary measurements}

With the aim of determining the baseline of STD, the demodulator was initially tested with $A_{\mathrm{s}}=0$. In these conditions, the period measurement of a triangular signal with $f_{0}=1 \mathrm{kHz}$ had an STD $\approx 50 \mathrm{~ns}$, which can be ascribed to the inherent noise of the two signals under comparison and to the comparator noise. In addition, the histogram of the period measurements was clearly Gaussian. 


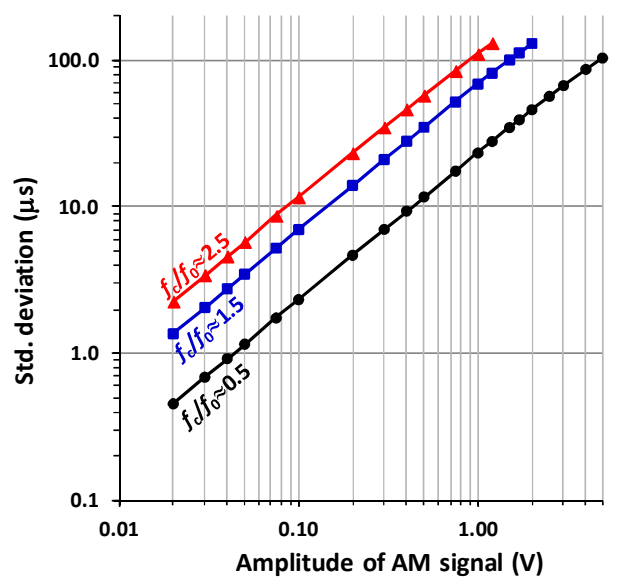

Fig. 8. Experimental values of STD of the period samples versus the peak-to-peak amplitude of the AM signal for $f_{\mathrm{c}} / f_{0} \approx 0.5,1.5$, and 2.5 .

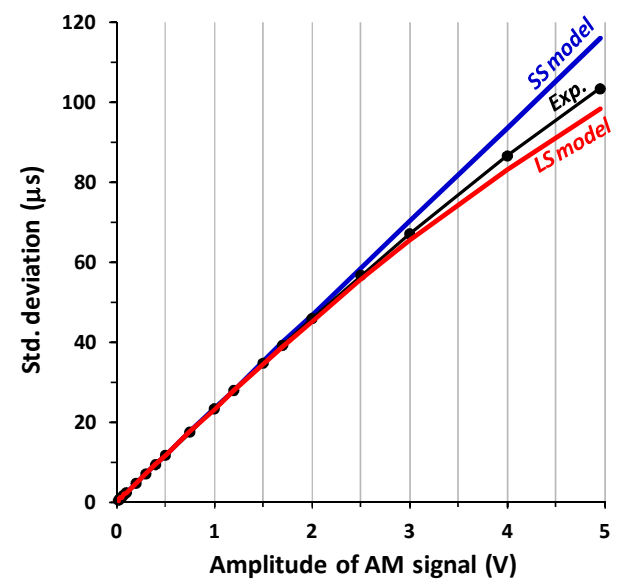

Fig. 9. Theoretical and experimental values of STD versus the peakto-peak amplitude of the AM signal for $f_{\mathrm{c}} / f_{0} \approx 0.5$.

For $A_{\mathrm{s}} \neq 0$ and $f_{\mathrm{c}} / f_{0}=m$, the STD was also around $50 \mathrm{~ns}$ and the histogram was also Gaussian regardless of the value of $A_{\mathrm{s}}$, which was tested up to $5 \mathrm{~V}$. Very similar results were obtained when $f_{\mathrm{c}} / f_{0}$ was exactly equal to $m+0.5$ since the alias component had a frequency of $0 \mathrm{~Hz}$. Therefore, these ratios cannot be employed to extract the amplitude of the AM signal, as already suggested in Section III. The main difference between these two cases was that the histogram was centered at the ideal value (i.e. $T_{0}$ ) when $f_{\mathrm{c}} / f_{0}=m$, but there was a bias when $f_{\mathrm{c}} / f_{0}=m+0.5$ that depended on $\varphi$, as predicted by (A.2).

\section{C.Aliasing}

The demodulator was then tested for $A_{\mathrm{s}} \neq 0$ and $f_{\mathrm{c}} / f_{0}=(m+0.5)(1+\Delta)$. Fig. 7 shows, for instance, the period samples obtained for $A_{\mathrm{s}}=500 \mathrm{mV}, m=1$, and $\Delta=-0.67 \%$ and $0.34 \%$, which involve $f_{\mathrm{c}} / f_{0}=1.49$ and 1.505 , respectively. The waves reconstructed from the samples in Fig. 7 correspond to an alias component of $10 \mathrm{~Hz}$ and $5 \mathrm{~Hz}$, respectively, which agrees with $f_{c} \cdot|\Delta|$. These two waves were not centered at the same value in the y-axis because they correspond to different values of $T_{0}$, to be precise: $T_{0}=993 \mu \mathrm{s}$ and $1003 \mu \mathrm{s}$ for $f_{\mathrm{c}} / f_{0}=1.49$ and 1.505 , respectively.

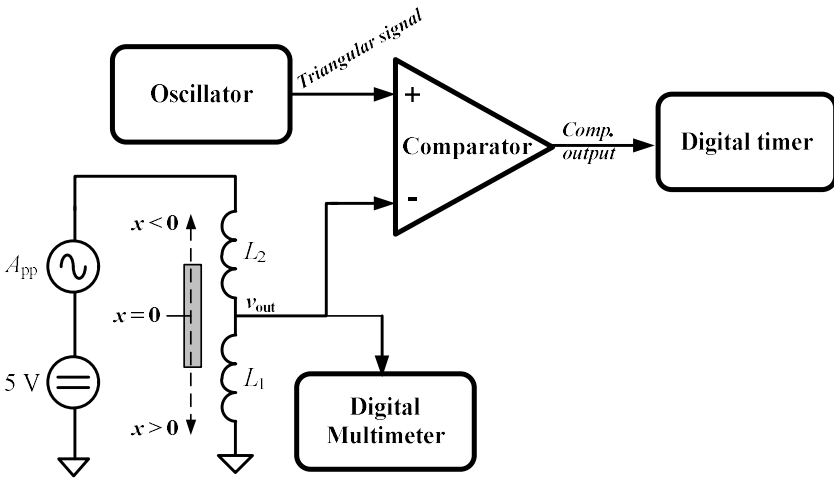

Fig. 10. Timer-based demodulator applied to measure an inductive displacement sensor. The differential topology of the sensor is configured as an AC voltage divider.

\section{D.STD vs signal amplitude}

The amplitude and, hence, the STD of the alias component represented in Fig. 7 clearly depended on $A_{\mathrm{s}}$. This is shown in more detail in Fig. 8 on a $\log$-log scale for $f_{\mathrm{c}} / f_{0} \approx 0.5,1.5$, and 2.5 ; the ratios were selected so that the alias component had a frequency of $10 \mathrm{~Hz}$. For the three cases, the higher the amplitude of the AM signal, the higher the STD, which is the essence of the proposed demodulator. As for the operating range, aberrant measurements were obtained when $A_{\mathrm{s}}>2.0 \mathrm{~V}$ for $f_{\mathrm{c}} / f_{0} \approx 1.5$, and when $A_{\mathrm{s}}>1.2 \mathrm{~V}$ for $f_{\mathrm{c}} / f_{0} \approx 2.5$, which agrees with (13). No aberrant measurements were seen for $f_{\mathrm{c}} / f_{0} \approx 0.5$ since the values of $A_{\mathrm{s}}$ under test were lower than the maximum value predicted by (13).

\section{E. Linearity}

The non-linearity expected at high values of $A_{\mathrm{s}}$ can be better observed by representing STD versus $A_{\mathrm{s}}$ on a linear scale, as shown in Fig. 9 for $f_{\mathrm{c}} / f_{0} \approx 0.5$. Besides the experimental data already represented in Fig. 8, Fig. 9 also shows the STD calculated by both the small-signal model (7) and the largesignal model (11), identified as "SS model" and "LS model", respectively. The SS model satisfactorily predicts the response of the demodulator up to $A_{\mathrm{s}} \approx 2 \mathrm{~V}$, whereas the LS model is able to predict the non-linearity obtained at higher values of $A_{\text {s. }}$. The discrepancy between the experimental data and the LS model shown in Fig. 9 can be ascribed to limitations of the model. Note that a second-order Taylor-series approximation is assumed in (8), and that (9) is valid when $f_{c} / f_{0}$ is exactly equal to $m+0.5$.

\section{F. Discussion}

With regard to the selection of $m$ when the demodulator operates at $f_{\mathrm{c}} / f_{0}=(m+0.5)(1+\Delta)$, the following issues should be considered. A low value of $m$ enables the demodulation of signals with higher amplitude, but with a non-linearity response at these high values, as shown in Figs. 8 and 9. A low value of $m$ also involves a higher value of the sampling frequency, as indicated by (14), which means that a higher number of samples can be taken in one period of the alias component. The higher the number of samples, the better the estimation of STD, but the longer the processing time. On the other hand, a high value of $m$ involves a triangular signal of lower frequency that causes a higher value of STD, as shown 


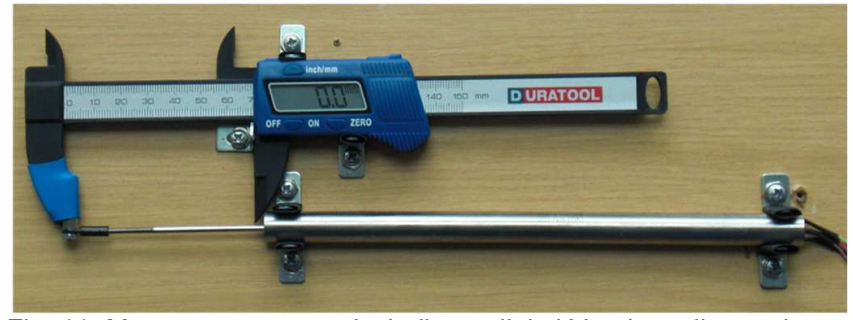

Fig. 11. Measurement setup including a digital Vernier caliper to know the actual position of the core inside the displacement sensor.

in Fig. 8, because the SR is smaller. Furthermore, in such a case, the (relative) uncertainty caused by the quantization of the timer is smaller.

\section{Application to AN INDUCtive Sensor}

\section{A. Materials and method}

The proposed timer-based demodulator has been applied to measure an inductive displacement sensor with a differential topology, as shown in Fig. 10. This was assembled using the two secondary coils of a commercial AC linear variable differential transformer (LVDT), model M-12 65 from Measurement Specialties; the primary coil of the LVDT was not employed [29]. The sensor was excited by a waveform generator (Agilent 33210A) providing a sinusoidal signal superimposed on a DC level of $5 \mathrm{~V}$. This sinusoidal signal had a peak-to-peak amplitude of $A_{\mathrm{pp}}$ at $f_{\mathrm{c}}=1500 \mathrm{~Hz}$. The output of the sensor $\left(v_{\text {out }}\right)$ had the same frequency but its amplitude was modulated by the position $(x)$ of the magnetic core, with a length of $60 \mathrm{~mm}$, inside the sensor. The actual position was monitored by a digital Vernier caliper attached to the coreconnecting rod, as shown in Fig. 11. Since the two inductances $\left(L_{1}\right.$ and $L_{2}$ ) of the sensor had the same DC parasitic resistance and this was independent of $x$, the DC level of $v_{\text {out }}$ was $2.5 \mathrm{~V}$, thus facilitating the comparison with the triangular signal. The rest of the electronic instrumentation employed in Fig. 10 was the same indicated in Section IV.A.

The sensor output was measured by both a digital multimeter (Agilent 34410A) in AC voltage mode and the proposed demodulator at different positions of the core. Two cases were tested: (a) $A_{\mathrm{pp}}=5 \mathrm{~V}$ and $f_{\mathrm{c}} / f_{0}=0.5 \cdot(1-0.67 \%)$, and (b) $A_{\mathrm{pp}}=2 \mathrm{~V}$ and $f_{\mathrm{c}} / f_{0}=1.5 \cdot(1-0.67 \%)$. The value of $A_{\mathrm{pp}}$ was selected to be smaller than that provided by (13) to avoid aberrant measurements, whereas the value of $\Delta$ was chosen to have an alias component of $10 \mathrm{~Hz}$.

\section{B. Experimental results}

The two cases indicated before provided the results represented in Figs. 12 and 13, respectively, in the range of $\pm 30 \mathrm{~mm}$ around the center of the sensor. In both figures, the output of the timer-based demodulator (i.e. the STD of the period samples) was able to accurately follow the amplitude change of the AM signal caused by the change of the sensor position. Although the amplitude to be demodulated was lower in Fig. 13, the resulting STD was higher than in Fig. 12 since the SR of the triangular signal was lower. The linearity was then evaluated by representing STD versus the amplitude of $v_{\text {out }}$, thus obtaining a maximum non-linearity error (NLE) of

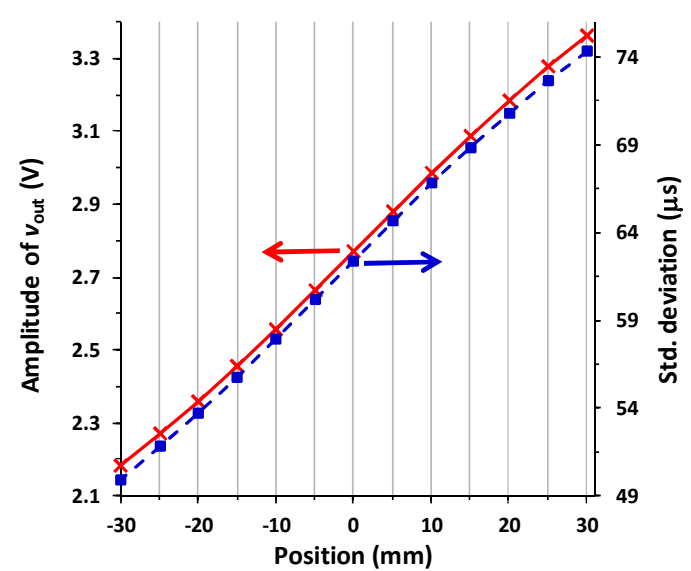

Fig. 12. Peak-to-peak amplitude of the sensor output signal and STD measured by the timer-based demodulator at different positions of the core inside the sensor, when $A_{\mathrm{pp}}=5 \mathrm{~V}$ and $f_{\mathrm{c}} / f_{0} \approx 0.5$

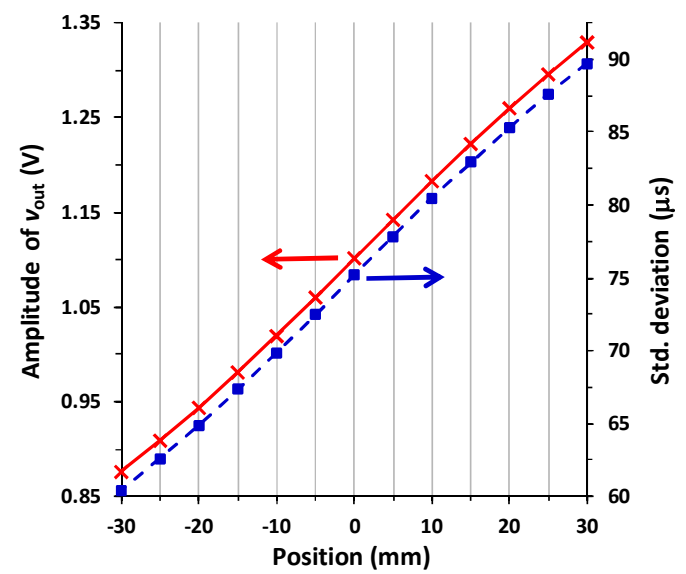

Fig. 13. Peak-to-peak amplitude of the sensor output signal and STD measured by the timer-based demodulator at different positions of the core inside the sensor, when $A_{\mathrm{pp}}=2 \mathrm{~V}$ and $f_{\mathrm{c}} / f_{0} \approx 1.5$.

$0.7 \%$ and $0.5 \%$ full-scale span (FSS) in cases (a) and (b), respectively.

The measurement resolution in Figs. 12 and 13 can be computed by dividing the output span by the residual value of STD of $50 \mathrm{~ns}$ found in Section IV.B. The result is a resolution of 9 bits for a measuring time of $100 \mathrm{~ms}$ (i.e. $1 / 10 \mathrm{~Hz}$ ), which corresponds to $0.1 \mathrm{~mm}$ in a $\pm 30 \mathrm{~mm}$ range. Such a resolution could be improved by: (a) increasing the frequency of the crystal oscillator of the timer so as to have a residual value lower than $50 \mathrm{~ns}$, and (b) employing an amplifier between the sensor output and the comparator in order to increase the span of the signal amplitude. This, however, could cause an increment of the non-linearity.

\section{C.Discussion}

The circuit shown in Fig. 10 was mainly intended to prove the feasibility of the novel demodulation technique with a commercial sensor, but it was not optimized to show its benefits in terms of current consumption. Note that the static current consumption of the inductive sensor was of several milliamperes and, hence, the fact of using a TDC-based circuit instead of an ADC-based circuit offers a small improvement in the overall current consumption of the circuit. In order to 
better show the advantages of the timer-based demodulator in terms of current consumption, it would be preferable, for instance, to demodulate the signal coming from a capacitive sensor that does not consume static power.

According to Section V.B, the proposed demodulator has an NLE $\left(0.5 \%\right.$ FSS that corresponds to an $\mathrm{R}^{2}$ value of 0.9999$)$ that is comparable to and even better than those presented in the literature, for instance: an NLE of $1.4 \%$ FSS in [2] and an $\mathrm{R}^{2}$ value of 0.998 in [11]. Commercial monolithic signal conditioners for inductive sensors with a differential topology, such as the AD598 from Analog Devices, offer an NLE of several tenths of $1 \%$, which is also similar to that obtained here. The duration of the measurement, which is in the range of tens or hundreds of millisecond, is the major drawback of the proposed demodulator, thus limiting the measurement to slowly varying magnitudes. This also happens in the circuits for resistive displacement sensors recently suggested in $[35,36]$ with a conversion time of $100 \mathrm{~ms}$ and $1.2 \mathrm{~s}$, respectively, which have been proposed for automobile applications. Such a long conversion time is not a handicap either for the demodulation of AM signals coming from capacitive sensors that monitor slow magnitudes such as relative humidity [25] and soil water content [37].

\section{CONCLUSION}

This work has gone a step further in the field of sensor electronic interfaces by employing a digital timer to demodulate AM sinusoidal signals. It has been theoretically and experimentally proved that the amplitude of AM signals can be extracted by measuring -with a digital timer- the period of a reference triangular signal that is altered by the AM signal itself. However, in order to have the expected output, an appropriate relation between the carrier frequency of the AM signal and the frequency of the triangular signal has to be selected. The application of the proposed demodulator to read the signal coming from an inductive displacement sensor excited at $1500 \mathrm{~Hz}$ has shown the following features: a maximum NLE of $0.5 \%$ FSS, a resolution of 9 bits, and an overall measuring time of $100 \mathrm{~ms}$. The main advantage of the proposed demodulator is the fact that the demodulation is carried out directly in the digital domain without using an ADC, with the corresponding benefits in terms of current consumption and die area. However, this is at the expense of a long conversion time. The demodulation of AM signals with carrier suppression and the use of two digital timers operating in parallel will be investigated in the next future.

\section{APPENDIX}

For the case of interest in which $f_{\mathrm{c}} / f_{0}=m+0.5$, the resulting deviation evolves as indicated by (7). If this sinusoidal signal is sampled every $T_{\mathrm{s}}\left(=1 / f_{\mathrm{s}}\right)$, the result is a discrete-time signal that can be expressed as

$$
e_{\mathrm{T}}[n]=e_{\mathrm{T}}\left(n T_{\mathrm{s}}\right)=\frac{A_{\mathrm{s}}}{\mathrm{SR}} \sin \left(2 \pi f_{\mathrm{c}} n T_{\mathrm{s}}+\varphi\right)
$$

where $n$ is the sample number. Assuming $f_{\mathrm{s}}=f_{0} / 2,(\mathrm{~A} .1)$ is simplified to

$$
e_{\mathrm{T}}[n]=\frac{A_{\mathrm{s}}}{\mathrm{SR}} \sin \varphi
$$

which shows that all samples have the same value, thus generating an alias component at $0 \mathrm{~Hz}$. However, if the demodulator operates at $f_{\mathrm{c}} / f_{0}=(m+0.5)(1+\Delta)$, then (6) can be rewritten as

$$
e_{\mathrm{T}}(t)=\frac{A_{\mathrm{s}}}{\mathrm{SR}} \cos (\pi \varepsilon) \sin \left(2 \pi f_{\mathrm{c}} t+\varphi-\pi \varepsilon\right)
$$

where $\varepsilon=\Delta(m+0.5)$. If (A.3) is now sampled at $f_{\mathrm{s}}=f_{0} / 2$, then the resulting discrete-time signal is

$$
e_{\mathrm{T}}[n]=\frac{A_{\mathrm{s}}}{\mathrm{SR}} \cos (\pi \varepsilon) \sin [\pi \varepsilon(4 n-1)+\varphi]
$$

which is periodic every $n_{\mathrm{s}}$ samples, where $n_{\mathrm{s}}=1 /(2|\varepsilon|)$. This involves an alias component with a frequency equal to

$$
f_{\text {alias }}=\frac{f_{\mathrm{s}}}{n_{\mathrm{s}}}=f_{\mathrm{c}} \frac{|\Delta|}{1+\Delta} \approx f_{\mathrm{c}}|\Delta|
$$

where $f_{\mathrm{s}}$ is defined in (14). For example, if $f_{\mathrm{c}}=1500 \mathrm{~Hz}$ and $\Delta=-0.67 \%$, then $\varepsilon=-3.33 \cdot 10^{-3}$ and $-9.99 \cdot 10^{-3}$ for $m=0$ and 1 , respectively, involving 150 and 50 samples to have a complete period of the alias component at $10 \mathrm{~Hz}$.

\section{REFERENCES}

[1] S. Nihtianov, "Measuring in the subnanometer range: capacitive and eddy current nanodisplacement sensors," IEEE Ind. Electron. Magazine, vol. 8, no. 1, pp. 6-15, March 2014.

[2] N. Mandal, B. Kumar, R. Sarkar, and S. C. Bera, "Design of a flow transmitter using an improved inductance bridge network and rotameter as sensor," IEEE Trans. Instrum. Meas., vol. 63, no. 12, pp. 3127-3136, Dec. 2014

[3] E. Sardini, M. Serpelloni, and V. Pasqui, "Wireless wearable T-shirt for posture monitoring during rehabilitation exercises," IEEE Trans. Instrum. Meas., vol. 64, no. 2, pp. 439-448, Feb. 2015.

[4] S. Chattopadhyay and S. C. Bera, "Modification of the Maxwell-Wien bridge for accurate measurement of a process variable by an inductive transducer," IEEE Trans. Instrum. Meas., vol. 59, no. 9, pp. 2445-2449, Sept. 2010.

[5] S. C. Bera, N. Mandal, and R. Sarkar, "Study of a pressure transmitter using an improved inductance bridge network and Bourdon tube as transducer," IEEE Trans. Instrum. Meas., vol. 60, no. 4, 1453-1460, April 2011.

[6] P. Kumar, B. George, and J. Kumar, "A simple signal conditioning scheme for inductive sensors," in Proc. Int. Conf. Sensing Technol., pp. $512-515,2013$

[7] E. G. Bakhoum and M. H. M. Cheng, "High-sensitivity inductive pressure sensor," IEEE Trans. Instrum. Meas., vol. 60, no. 8, pp. 29602966, August 2011.

[8] W. Q. Yang, "A self-balancing circuit to measure capacitance and loss conductance for industrial transducer applications," IEEE Trans. Instrum. Meas., vol. 45, no. 6, pp. 955-958, Dec. 1996.

[9] G. Langfelder, G. Laghi, P. Minotti, A. Tocchio, and A. Longoni, "Offresonance low-pressure operation of Lorentz force MEMS magnetometers," IEEE Trans. Ind. Electron., vol. 61, no. 12, pp. 71247130, Dec. 2014 
[10] C. Kolle and P. O. Leary, "Low-cost, high-precision measurement system for capacitive sensors," Meas. Sci. Technol., vol. 9, pp. 510-517, 1998.

[11] M. R. Haider, M. R. Mahfouz, S. K. Islam, S. A. Eliza, W. Qu, and E. Pritchard, "A low-power capacitance measurement circuit with high resolution and high degree of linearity," in Proc. Midwest Symp. Circuits Syst, pp. 261-264, 2008 .

[12] S. M. Zhak, M. W. Baker, and R. Sarpeshkar, "A low-power wide dynamic range envelope detector," IEEE J. Solid-State Circuits, vol. 38, no. 10 , pp. $1750-1753$, Oct. 2003.

[13] J. P. Alegre, S. Celma, B. Calvo, and J. M. Garcia del Pozo, "Design of a novel envelope detector for fast-settling circuits," IEEE Trans. Instrum. Meas., vol. 57, no. 1, pp. 4-9, Jan. 2008.

[14] J. Aguirre, D. García-Romeo, N. Medrano, B. Calvo, and S. Celma, "Square-signal-based algorithm for analog lock-in amplifiers," IEEE Trans. Ind. Electron., vol. 61, no. 10, pp. 5590-5598, Oct. 2014.

[15] Z. Cui, H. Wang, and W. Yin, "Electrical capacitance tomography with differential sensor," IEEE Sens. J., vol. 15, no. 9, pp. 5087-5094, 2015

[16] S. Henzler, Time-to-digital converters, Dordrecht: Springer, 2010.

[17] L. Bengtsson, "Embedded measurement systems," Ph.D. dissertation, Dep. Physics, Univ. Gothenburg, Gothenburg, Sweden, 2013.

[18] Y. J. An, K. Ryu, D. H. Jung, S. H. Woo, and S. O. Jung, "An energy efficient time-domain temperature sensor for low-power on-chip thermal management," IEEE Sensors J., vol. 14, no. 1, pp. 104-110, Jan. 2014.

[19] Y. L. Lo and Y. T. Chiu, "A high-accuracy, high-resolution, and lowcost all-digital temperature sensor using a voltage compensation ring oscillator," IEEE Sensors J., vol. 16, no. 1, pp. 43-52, Jan. 2016.

[20] S. Wang and C. Dehollain, "Design and implementation of a rail-to-rail 460-kS/s 10-bit SAR ADC for the power-efficient capacitance measurement," IEEE Trans. Instrum. Meas., vol. 64, no. 4, 888-901, April 2015.

[21] A. L. Aita, M. A. P. Pertijs, K. A. A. Makinwa, J. H. Huijsing, and G. C. M. Meijer, "Low-power CMOS smart temperature sensor with a batchcalibrated inaccuracy of $\pm 0.25^{\circ} \mathrm{C}( \pm 3 \sigma)$ from $-70^{\circ} \mathrm{C}$ to $130^{\circ} \mathrm{C}, "$ IEEE Sensors J., vol. 13, no. 5, pp. 1840-1848, May 2013.

[22] F. Reverter, J. Jordana, M. Gasulla, and R. Pallàs-Areny, "Accuracy and resolution of direct resistive sensor-to-microcontroller interfaces," Sens. Actuators A, vol. 121, pp. 78-87, 2005.

[23] F. Reverter and Ò. Casas, "Interfacing differential resistive sensors to microcontrollers: a direct approach," IEEE Trans. Instrum. Meas., vol. 58 , no. 10 , pp. 3405-3410, Oct. 2009

[24] O. Oballe-Peinado, F. Vidal-Verdú, J. A. Sánchez-Durán, J. CastellanosRamos, and J. A. Hidalgo-López, "Accuracy and resolution analysis of a direct resistive sensor array to FPGA interface," Sensors, vol. 16, no. 2, pp. 181-196, 2016.

[25] F. Reverter and Ò. Casas, "Direct interface circuit for capacitive humidity sensors," Sens. Actuators A, vol. 143, pp. 315-322, 2008.

[26] F. Reverter and Ò. Casas, "Interfacing differential capacitive sensors to microcontrollers: a direct approach," IEEE Trans. Instrum. Meas., vol. 59, no. 10 , pp. 2763-2769, Oct. 2010

[27] J. Pelegrí-Sebastiá, E. García-Breijo, J. Ibáñez, T. Sogorb, N. LaguardaMiro, and J. Garrigues, "Low-cost capacitive humidity sensor for application within flexible RFID labels based on microcontroller systems," IEEE Trans. Instrum. Meas., vol. 61, no. 2, pp. 545-553, Feb. 2012.

[28] Z. Kokolanski, J. Jordana, M. Gasulla, V. Dimcev, and F. Reverter, "Direct inductive sensor-to-microcontroller interface circuit," Sens. Actuators A, vol. 224, pp. 185-191, 2015.

[29] N. Ramadoss and B. George, "A simple microcontroller based digitizer for differential inductive sensors," in Proc. IEEE Int. Instrum. Meas. Technol. Conf., pp. 148-153, 2015.

[30] L. Bengtsson, "Direct analog-to-microcontroller interfacing," Sens. Actuators A, vol. 179, pp. 105-113, 2012.

[31] M. R. Nabavi and S. Nihtianov, "Eddy-current sensor interface for advanced industrial applications," IEEE Trans. Ind. Electron., vol. 58, no. 9, pp. 4414-4423, Sept. 2011.

[32] F. Reverter and R. Pallàs-Areny, "Uncertainty reduction techniques in microcontroller-based time measurements," Sens. Actuators A, vol. 127 pp. 74-79, 2006.

[33] F. Reverter, M. Gasulla, and R. Pallàs-Areny, "Analysis of interference effects on period-to-digital conversions," Meas. Sci. Technol., vol. 16, pp. 2261-2264, 2005.
[34] J. R. Westra, C. J. M. Verhoeven, and A. H. M. van Roermund, Oscillators and Oscillator Systems. Classification, Analysis and Synthesis, Boston: Kluwer Academic Publishers, 1999.

[35] S. Rana, B. George, and V. J. Kumar, "Sigma-delta digital converter suitable for a resistive displacement sensor with a floating slide," IEEE Trans. Instrum. Meas., vol. 65, no. 3, pp. 502-509, Mar. 2016.

[36] S. V. Thathachary, B. George, and V. J. Kumar, "Digital converter for a contactless displacement sensor," IEEE Trans. Instrum. Meas., vol. 64, no. 8, pp. 2155-2164, Aug. 2015.

[37] E. F. da Costa, N. E. de Oliveira, F. J. O. Morais, P. Carvalhaes-Dias, L. F. C. Duarte, A. Cabot, and J. A. S. Dias, "A self-powered and autonomous fringing field capacitive sensor integrated into a micro sprinkler spinner to measure soil water content," Sensors, vol. 17, no. 3 , $575,2017$. 